\title{
Quarta idade e velhice extrema: substituição da polifarmácia por um comprimido de hidromorfona de liberação controlada para o controle da dor crônica*
}

\author{
Fourth age and extreme old age: replacement of polypharmacy by a controlled-release hydromorphone \\ tablet to control chronic pain
}

Gabriela Rocha Lauretti ${ }^{1}$, Anita Leocádia de Mattos²

* Recebido do Hospital das Clínicas da Faculdade de Medicina de Ribeirão Preto da Universidade de São Paulo. Ribeirão Preto, SP.

\section{RESUMO}

JUSTIFICATIVA E OBJETIVOS: Cerca de 4,5 milhões de pessoas terão idade superior a 80 anos até 2020 . Frequentemente, esta população necessita de polifarmácia para o controle da dor. O objetivo deste estudo foi avaliar a eficácia, custo e segurança da administração única, diária de hidromorfona de liberação controlada em pacientes com idade $>80$ anos.

MÉTODO: Oito pacientes (82 a 89 anos, quarta idade), e dois pacientes (93 e 99 anos, velhice extrema) foram avaliados. Todos utilizavam opioides, antidepressivos, anti-inflamatórios não esteroides, paracetamol e anticonvulsivantes para o controle da dor. Todos os fármacos com finalidade analgésica foram substituídos por um comprimido diário de $8 \mathrm{mg}$ de hidromorfona de liberação controlada (OROS). Dor e efeitos adversos foram avaliados.

RESULTADOS: O número de comprimidos diários para controle da dor diminuiu de 6-7 para um de hidromorfona. Houve diminuição da sensação de mal estar gástrico matinal, secundária à ingestão de grande número de comprimidos. Não foram relatadas náusea ou indisposição. Não foi relatada sonolência diária, sendo que os pacientes referiram melhor padrão de sono.

CONCLUSÃO: A substituição da polifarmácia por um

1. Médica Anestesiologista; Professora Associada da Faculdade de Medicina de Ribeirão Preto da Universidade de São Paulo. Ribeirão Preto, SP, Brasil.

2. Médica Anestesiologista; Professora Doutora da Faculdade de Medicina de Ribeirão Preto da Universidade de São Paulo. Ribeirão Preto, SP, Brasil.

Endereço para correspondência:

Dra. Gabriela Rocha Lauretti

Rua Maestro Joaquim Rangel, 644 - Bairro Alto da Boa Vista 14025-610 Ribeirão Preto, SP.

E-mail: grlauret@fmrp.usp.br comprimido diário de hidromorfona melhorou o padrão analgésico, com baixa incidência de efeitos adversos em pacientes idosos, portadores de dor crônica, podendo ser considerada como uma boa alternativa para o controle da dor e a melhora da qualidade de vida desta população. Descritores: Analgesia, Dor, Hidromorfona, Idoso.

\section{SUMMARY}

BACKGROUND AND OBJECTIVES: Approximately 4.5 million people will have more than 80 years of age by 2020 . Very often, this population needs polypharmacy to control pain. This study aimed at evaluating the effectiveness, cost and safety of a single daily dose of controlled-release hydromorphone in patients aged $>80$ years.

METHOD: Eight patients ( 82 to 89 years old, fourth age) and two patients (93 and 99 years old, extreme old age) were evaluated. All were under opioids, antidepressants, non-steroid anti-inflammatory drugs, paracetamol and anticonvulsants to control pain. All analgesic drugs were replaced by a daily $8 \mathrm{mg}$ tablet of controlled-release hydromorphone (OROS). Pain and adverse effects were evaluated.

RESULTS: The number of daily tablets to control pain was decreased from 6-7 to one hydromorphone tablet. The morning gastric malaise sensation, secondary to the ingestion of a large number of tablets has decreased. There were no nausea or distemper. There were no reports on daily sleepiness and patients have referred a better sleeping pattern.

CONCLUSION: Replacing polypharmacy by a daily hydromorphone tablet has improved the analgesic pattern, with low incidence of adverse effects in elderly patients with chronic pain and it may be considered a good alternative to control pain and improve quality of life of this population. Keywords: Analgesia, Elderly, Hydromorphone, Pain. 


\section{INTRODUÇÃO}

Idoso é um termo que indica uma pessoa com uma vivência traduzida em muitos anos. Em geral, a literatura classifica, didaticamente, as pessoas acima de 65 anos como idosos e participantes da "terceira idade". Na década de 1960, apenas 5\% da população eram representados por idosos. Entretanto, devido aos avanços científicos e a melhora da qualidade de vida, considera-se atualmente a existência de uma "quarta idade" que englobaria pessoas com 80 anos ou mais e da categoria "velhice extrema", a qual englobaria pessoas com idade superior a 90 anos. De acordo com algumas estimativas, esta faixa etária alcançará uma representatividade com cerca de 4,5 milhões pessoas até 2020 .

O aumento do número de idosos também tem sido acompanhado por um acréscimo significativo nos anos de vida da população brasileira. A esperança de vida, que era em torno de 33,7 anos em 1950/1955, passou para 50,99 em 1990, chegou até 66,25 em 1995 e deverá alcançar 77,08 em 2020/2025, conforme dados da Organização Mundial de Saúde (OMS, 2010).

O dado mais preocupante é que este tempo de vida não é alcançado de forma satisfatória e sem graves problemas; ao contrário, estes seres humanos apresentam um quadro de dor secundária à degeneração temporal inevitável do organismo, somada à dor secundária de doenças da juventude, como diabetes, artrose, dor miofascial, entre outras. Inevitavelmente, estes pacientes utilizam polifarmácia para o controle da dor crônica e das comorbidades associadas, como hipertensão arterial, diabetes, artrose, miopatias, etc. ${ }^{1,2}$.

O objetivo deste estudo foi avaliar a eficácia, custo e segurança da administração diária de um comprimido de hidromorfona de liberação controlada (OROS) em pacientes portadores de dor crônica ${ }^{3}$, com idade superior a 80 anos, insatisfeitos com a polifarmácia analgésica.

\section{MÉTODO}

Após aprovação pelo Comitê de Ética em Pesquisa do Hospital das Clínicas da Faculdade de Medicina de Ribeirão Preto da Universidade de São Paulo (projeto $\mathrm{n}^{\mathrm{o}}$ CAAE-0366.0.004.000-10) e após consentimento formal, 10 pacientes fizeram parte do protocolo de estudo de avaliação de velhice extrema e quarta idade. Oito pacientes apresentavam idade entre 82 e 89 anos (quarta idade) e dois pacientes apresentavam respectivamente 93 e 99 anos (velhice extrema).

Todos os pacientes eram orientados e apresentavam outras comorbidades como hipertensão arterial sistêmica, insufi- ciência cardíaca leve a moderada, diabetes tipo II, dor crônica tipo neuropática e somática, com comprometimento articular, miofascial e neuropático lombar, cervical ou neuropatia pós-herpética. Todos os pacientes faziam uso regular de polifarmácia para controle das comorbidades e da dor, incluindo tramadol, codeína, morfina, anticonstipantes, antidepressivos, anti-inflamatórios não esteroides (AINES), paracetamol e anticonvulsivantes.

Todos os fármacos com finalidade analgésica, com exceção ao antidepressivo, foram substituídos por um comprimido de $8 \mathrm{mg}$ de hidromorfona de liberação controlada, pela manhã. Dor e efeitos adversos foram avaliados diariamente por 30 dias; utilizando-se a escala analógica visual (EAV) de zero a $10 \mathrm{~cm}$, sendo zero ausência de dor/náusea, sedação, variando até $10 \mathrm{~cm}$, correspondente ao pior sintoma possível e imaginável. O grau de satisfação inicial e final quanto ao uso diário de medicamentos foi avaliado através da EAV (0-10 $\mathrm{cm})$ sendo zero equivalente a "completamente satisfeito" variando até $10 \mathrm{~cm}$, equivalente a "completamente insatisfeito".

Cada paciente atuou como seu próprio controle. Dados referentes ao controle da dor (EAV), número de comprimidos diários necessários para o controle da dor, satisfação e dos efeitos adversos foram avaliados pelos testes Mann-Whitney e Wilcoxon pareado. O valor de $\mathrm{p}<0,05$ foi considerado significante.

\section{RESULTADOS}

A tabela 1 descreve os dados demográficos, as comorbidades e os fármacos analgésicos utilizados pelos pacientes antes da administração da hidromorfona. Os valores numéricos referentes à intensidade da dor avaliados pela escala analógica visual $(0-10 \mathrm{~cm})$ antes e após a introdução diária do comprimido de hidromorfona de liberação controlada e o grau de satisfação estão descritos na tabela 2 .

Inicialmente todos os pacientes usavam regularmente 5 a 7 comprimidos diários para controle da dor, sendo a dor classificada diariamente como EAV 4 a $6 \mathrm{~cm}$. Todos os fármacos analgésicos foram substituídos pela administração de um comprimido de hidromorfona de $8 \mathrm{mg}$ pela manhã, e foi mantido o antidepressivo diário já prescrito. Após a introdução da hidromorfona, a classificação da dor foi EAV 0 a $2 \mathrm{~cm}$ durante os 30 dias de avaliação $(\mathrm{p}>0,05)$. Apenas o paciente $\mathrm{n}^{\mathrm{o}} 5$; portador de neoplasia, com dor nociceptiva visceral e somática, necessitou do aumento da dose de 8 para 16 mg diários na segunda semana de avaliação.

Efeitos adversos incluíram a permanência da constipação, o que era auxiliado com dieta rica em fibras, mag- 
Tabela 1 - Dados demográficos da população avaliada.

\begin{tabular}{|c|c|c|c|c|c|c|}
\hline Paciente & Idade & Sexo & Outras Comorbidades & Estatura & Peso & $\begin{array}{l}\text { Número Diário de } \\
\text { Comprimidos Analgésicos }\end{array}$ \\
\hline 1 & 82 & $\mathrm{~F}$ & $\begin{array}{l}\text { HAS, insuficiência } \\
\text { pulmonar, gota, artrose }\end{array}$ & 162 & 78 & $\begin{array}{l}\text { Opioide (2) } \\
\text { Paracetamol (2) } \\
\text { Antidepressivo (1) } \\
\text { Anticonvulsivante (1) } \\
\text { AINE (1) }\end{array}$ \\
\hline 2 & 82 & M & Depressão, HAS, artrose & 167 & 68 & $\begin{array}{l}\text { Opioide (1) } \\
\text { Paracetamol (1) } \\
\text { Antidepressivo (1) } \\
\text { Anticonvulsivante (3) }\end{array}$ \\
\hline 3 & 83 & $\mathrm{~F}$ & $\begin{array}{l}\text { Diabetes, insuficiência } \\
\text { cardíaca, HAS, artrose }\end{array}$ & 155 & 58 & $\begin{array}{l}\text { Opioide (2) } \\
\text { Dipirona (2) } \\
\text { Antidepressivo (1) } \\
\text { AINE (1) }\end{array}$ \\
\hline 4 & 83 & $\mathrm{~F}$ & $\begin{array}{l}\text { Artrose, HAS, insuficiência } \\
\text { cardíaca, hipotireoidismo, } \\
\text { artrose }\end{array}$ & 156 & 56 & $\begin{array}{l}\text { Opioide (1) } \\
\text { Dipirona (1) } \\
\text { Antidepressivo (1) } \\
\text { Anticonvulsivante (2) }\end{array}$ \\
\hline 5 & 84 & $\mathrm{~F}$ & $\begin{array}{l}\text { Neoplasia vesical, HAS, } \\
\text { insuficiência urinária }\end{array}$ & 156 & 48 & $\begin{array}{l}\text { Opioide (4) } \\
\text { Dipirona (2) } \\
\text { Antidepressivo (1) }\end{array}$ \\
\hline 6 & 84 & M & $\begin{array}{l}\text { HAS, gota, dislipidemia, } \\
\text { artrose, obesidade }\end{array}$ & 166 & 72 & $\begin{array}{l}\text { Opioide (3) } \\
\text { Paracetamol (2) } \\
\text { Antidepressivo (1) } \\
\text { AINE (1) }\end{array}$ \\
\hline 7 & 88 & $\mathrm{~F}$ & $\begin{array}{l}\text { Artrose, HAS, diabetes-II, } \\
\text { varizes membros inferiores }\end{array}$ & 154 & 60 & $\begin{array}{l}\text { Opioide (2) } \\
\text { Paracetamol (2) } \\
\text { Antidepressivo (1) }\end{array}$ \\
\hline 8 & 89 & $\mathrm{~F}$ & $\begin{array}{l}\text { HAS, artrose, cegueira } \\
\text { senil, diabetes tipo II }\end{array}$ & 152 & 50 & $\begin{array}{l}\text { Opioide (2) } \\
\text { Paracetamol (2) } \\
\text { Antidepressivo (1) } \\
\text { Anticonvulsivante (1) }\end{array}$ \\
\hline 9 & 93 & $\mathrm{~F}$ & $\begin{array}{l}\text { Artrose, dislipidemia, } \\
\text { depressão }\end{array}$ & 155 & 54 & $\begin{array}{l}\text { Opioide (2) } \\
\text { Paracetamol (2) } \\
\text { Antidepressivo (2) }\end{array}$ \\
\hline 10 & 99 & $\mathrm{~F}$ & $\begin{array}{l}\text { HAS, glaucoma, artrose, } \\
\text { neuropatia pós-herpética }\end{array}$ & 150 & 49 & $\begin{array}{l}\text { Opioide (3) } \\
\text { Dipirona (2) } \\
\text { Antidepressivo (1) } \\
\text { Anticonvulsivante (1) }\end{array}$ \\
\hline
\end{tabular}

$\mathrm{M}=$ masculino; $\mathrm{F}$ = feminino; HAS = hipertensão arterial sistêmica; AINE = anti-inflamatório não esteroide 
Tabela 2 - Descrição do tipo, intensidade da dor e grau de satisfação do paciente.

\begin{tabular}{|c|c|c|c|c|c|}
\hline Paciente & $\begin{array}{l}\text { Tipos de Dor } \\
\text { Crônica }\end{array}$ & $\begin{array}{c}\text { Intensidade } \\
\text { Inicial da Dor }\end{array}$ & $\begin{array}{c}\text { Intensidade Final } \\
\text { da Dor }\end{array}$ & $\begin{array}{c}\text { Grau de Satisfação } \\
\text { Inicial }\end{array}$ & $\begin{array}{c}\text { Grau de Satisfação } \\
\text { Final }\end{array}$ \\
\hline 1 & $\begin{array}{l}\text { Nociceptiva } \\
\text { somática }\end{array}$ & 5 & 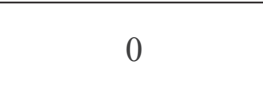 & 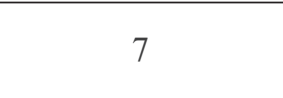 & 1 \\
\hline 2 & $\begin{array}{l}\text { Nociceptiva } \\
\text { somática }\end{array}$ & 4 & 2 & 8 & 2 \\
\hline 3 & $\begin{array}{l}\text { Nociceptiva } \\
\text { somática }\end{array}$ & 5 & 1 & 7 & 1 \\
\hline 4 & $\begin{array}{l}\text { Nociceptiva } \\
\text { somática } \\
\text { Nociceptiva }\end{array}$ & 4 & 0 & 8 & 0 \\
\hline 5 & $\begin{array}{l}\text { visceral e } \\
\text { somática }\end{array}$ & 6 & 1 & 8 & 2 \\
\hline 6 & $\begin{array}{l}\text { Nociceptiva } \\
\text { somática }\end{array}$ & 4 & 0 & 7 & 0 \\
\hline 7 & $\begin{array}{c}\text { Nociceptiva } \\
\text { somática e } \\
\text { neuropática } \\
\text { Nociceptiva }\end{array}$ & 5 & 2 & 9 & 2 \\
\hline 8 & $\begin{array}{l}\text { somática e } \\
\text { neuropática }\end{array}$ & 6 & 2 & 10 & 3 \\
\hline 9 & $\begin{array}{l}\text { Nociceptiva } \\
\text { somática }\end{array}$ & 4 & 0 & 7 & 0 \\
\hline 10 & $\begin{array}{c}\text { Nociceptiva } \\
\text { somática e } \\
\text { neuropática }\end{array}$ & 6 & 2 & 10 & 2 \\
\hline
\end{tabular}

nésio ou supositório de glicerina, dependendo do hábito do paciente. Houve diminuição da sensação de mal estar gástrico matinal, secundária a ingestão por via oral. Não foi relatada náusea ou indisposição. Não foi relatada sonolência diária, sendo que os pacientes referiram melhor padrão de sono (sono contínuo por pelo menos 5 horas), comparado com acordar frequente anterior ao tratamento com hidromorfona ( 4 a 5 vezes durante a noite).

Deve ser destacada a satisfação dos pacientes e familiares pela comodidade do tratamento com a substituição de 6 a 7 comprimidos por um único matinal $(\mathrm{p}<0,001)$, o que significou para o paciente uma alegria interna e contentamento, segundo seus relatos, com a sensação de estarem melhorando clinicamente, somente pelo fato da diminuição do número de fármacos analgésicos diários. A classificação pelos familiares acompanhantes foi de melhor satisfação e disposição dos pacientes $(\mathrm{p}<0,05)$.

\section{DISCUSSÃO}

A OMS classifica o envelhecimento em estágios, sendo idades entre 75 e 90 anos consideradas anciãos, e idade superior a 90 anos classificados como velhice extrema. Um crescimento mais acentuado foi percebido no grupo com 75 anos ou mais. Em 1996, eles representavam $23,5 \%$ da população de 60 anos ou mais. Dez anos depois, eles já eram $26,1 \%$. Infelizmente, a degeneração do organismo acompanha o processo do envelhecimento, resultando em pelo menos dois tipos de dor crônica, a dor neuropática, resultante da lesão do sistema nervoso periférico e central, e a dor somática, decorrente da degeneração do sistema musculoesquelético. A dor crônica passa a ser caracterizada como doença $a^{4}$, sendo imperioso seu tratamento, assim como outras comorbidades como hipertensão arterial sistêmica, diabetes, etc. O não tratamento da doença dor crônica pode inclusive ser responsável pelo número elevado de suicídios de pacientes idosos 5 .

De forma inédita, demonstrou-se neste estudo com pacientes com idade entre 82 e 99 anos que a administração diária de hidromorfona em pacientes portadores de dor crônica neuropática, somática e/ou visceral, usuários de opioides como codeína, morfina ou tramadol, associados à polifarmácia como AINES, anticonvulsivantes e analgé- 
sicos resultou em analgesia eficaz (EAV $<3 \mathrm{~cm}$ em todos os pacientes), desprovido de novos efeitos adversos.

Iniciou-se com a dose de $8 \mathrm{mg}$ diários, administrada a cada $24 \mathrm{~h}$ para pacientes, nos quais foram retirados os outros fármacos opioides e não opioides A hidromorfona de liberação lenta representa atualmente uma nova opção agonista opioide, com a comodidade da administração por via oral, a razão sugerida de conversão é de 5:1 equivalentes de morfina por hidromorfona ${ }^{6,7}$.

$\mathrm{O}$ efeito adverso que permaneceu foi constipação, o que não aborreceu os pacientes, pois já estavam habituados a lidar com este distúrbio intestinal. Entretanto, a ingestão de apenas um comprimido para o controle da dor ao invés de 5 a 7 diários, motivou os pacientes, resultou em analgesia eficaz, ausência de sono durante o dia, porém sono reconfortante durante a noite. Todos os pacientes e familiares optaram pela continuidade do tratamento da doença dor crônica com o uso de hidromorfona de liberação controlada (OROS) nesta população, sendo a administração de um monofármaco mais tolerável, para o paciente e economicamente viável quando comparado com a poli farmácia necessária para o controle da dor e dos seus efeitos adversos, sendo que apenas um dos pacientes necessitou de aumento da dose diária de 8 para $16 \mathrm{mg}$ de hidromorfona, sendo este paciente portador de dor neoplásica neuropática. Entretanto, mesmo a dose de $16 \mathrm{mg}$ diária foi eficaz e desprovida de efeitos adversos, estando o paciente em uso desta dose até a data atual $\left(45^{\circ}\right.$ dia).

Os resultados obtidos nesta investigação estão em acordo com outros estudos em idosos onde pacientes com idade média de 70 anos foram avaliados, onde os pacientes utilizaram hidromorfona durante 52 semanas, sendo eficaz e economicamente benéfico ${ }^{8}$, em acordo com os resultados do presente estudo. A formulação de liberação lenta proporciona analgesia estável, segura, sem a condição que comumente chama-se de "picos e vales", que favorece o aparecimento de efeitos adversos e um controle analgésico não ideal ${ }^{6,7}$.Outros autores relataram farmacocinética previsível em idosos, sendo a hidromorfona segura, previsível, eficaz e com baixa incidência de efeitos adversos, resultando em adição, melhora do padrão do sono e conforto posológico em idosos ${ }^{9}$.

No presente estudo o gasto mensal para controle da dor e dos efeitos adversos, como constipação, ansiedade, insônia e depressão variou entre $\mathrm{R} \$ 382,00$ e R $\$ 454,00$ (média de $\mathrm{R} \$ 35,00$ ao dia); o que tornou a administração da hidromorfona economicamente viável.

A substituição do uso de vários medicamentos diários para o controle da dor por um comprimido diário de 8 ou 16 mg de hidromorfona resultou em melhor padrão analgé- sico com baixa incidência de efeitos adversos em pacientes portadores de dor crônica somática e neuropática com idade superior a 82 anos (82 a 99 anos), representando uma alternativa viável e econônica ${ }^{3}$ para controle da dor e melhora da qualidade de vida nesta crescente população.

\section{CONCLUSÃO}

A substituição da polifarmácia por um comprimido diário de hidromorfona melhorou o padrão analgésico, com baixa incidência de efeitos adversos em pacientes idosos, portadores de dor crônica, podendo ser considerada como boa alternativa para o controle da dor e a melhora da qualidade de vida desta população.

\section{REFERÊNCIAS}

1. Zorba Paster R. Chronic pain management issues in the primary care setting and the utility of long-acting opioids. Expert Opin Pharmacother 2010;11(11):1823-33.

2. Pergolizzi J, Böger RH, Budd K, et al. Opioids and the management of chronic severe pain in the elderly: consensus statement of an International Expert Panel with focus on the six clinically most often used World Health Organization Step III opioids (buprenorphine, fentanyl, hydromorphone, methadone, morphine, oxycodone). Pain Pract 2008;8(4):287-313.

3. Carter NJ, Keating GM. OROS hydromorphone prolonged release: a review of its use in the management of chronic, moderate to severe pain. CNS Drugs 2010;24(4):337-61.

4. Pogatzki-Zahn E. Prevention and therapy of prolonged, chronic pain after surgery. Anasthesiol Intensivmed Notfallmed Schmerzther 2010;45(7-8):496-504.

5. Matusevich D. Qualitative analysis of eight suicide notes of elderly people. Vertex 2003;14(52):141-5.

6. Rizzo, JM, Nascimento MEC, Nascimento VC. Hidromorfona de liberação lenta: uma nova opção agonista opióide forte. Rev Dor 2010;11(1):68-73.

7. Serrano SC. Revisitando os analgésicos opioides. Editorial. Rev Dor 2010:11(1):3.

8. Alon E, Cachin C. Controlled release hydromorphone for visceral, somatic and neuropathic pain. Praxis 2010;99(5):283-91.

9. Lussier D, Richarz U, Finco G. Use of hydromorphone, with particular reference to the OROS formulation, in the elderly. Drugs Aging 2010;27(4):327-35.

Apresentado em 01 de novembro de 2010.

Aceito para publicação em 06 de setembro de 2011. 